\title{
Novel photon-counting detectors for free-space communication
}

\author{
Michael A. Krainak, Guan Yang,
}

Xiaoli Sun, Wei Lu, Scott Merritt,

NASA Goddard Space Flight Center, Greenbelt, MD USA 20771;

Jeff Beck

DRS Technologies, Network \& Imaging Systems, Dallas, Texas, USA 
Photon-counting detectors for

\section{AGENDA}

I. NASA applications/requirements

II. Silicon Geiger-mode avalanche photodiode array

III. Mercury Cadmium Telluride ( $\mathrm{HgCdTe})$ linear-mode avalanche photodiode array

IV. Summary 


\section{NASA-GSFC Single-Photon Counting Detectors NASA Goals for Near-Earth Free-Space Communication}

\begin{tabular}{|l|l|}
\hline Photon-counting wavelengths & Favor $1550 \mathrm{~nm}$, exploring $850 \mathrm{~nm}$ \\
\hline Detection efficiency: & $>10 \%$ \\
\hline Detector size: & $>200 \mu \mathrm{m}$ diameter \\
\hline 2-D arrays & Act as single detector w/high speed output \\
\hline Dark counts: & $<100 \mathrm{kcps}$ \\
\hline Maximum Count Rate: & $>1 \mathrm{Gbps}$ (through multiplexing) \\
\hline Electrical bandwidth: & $>1 \mathrm{GHz}$ \\
\hline Linearity: & $>98 \%$ fit \\
\hline Timing jitter: & $<100 \mathrm{ps}$ \\
\hline Afterpulsing & $<1 \%$ in $1 \mu \mathrm{s}$ \\
\hline & \\
\hline Operating temperature: & prefer thermo-electric cooler range \\
\hline Space-qualifiable: & rad-hard, reliable, overlight protection \\
\hline
\end{tabular}




\section{Sensl Silicon APD Array}

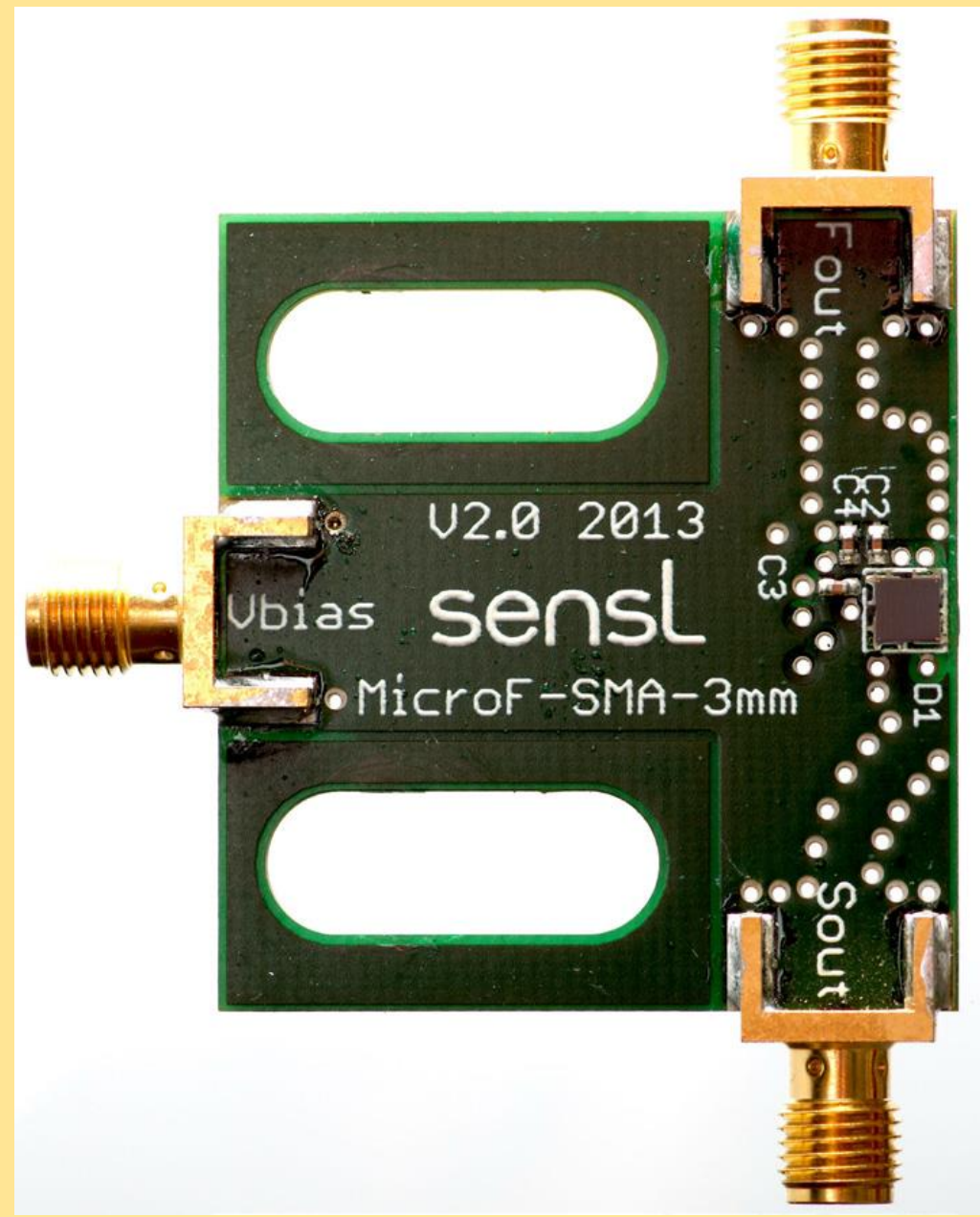

Detector: Sensl MicroFM-SMA10020

Lot \# 131218

Active Area: $1 \mathrm{~mm} \times 1 \mathrm{~mm}$

\# of Cells: 1144

Fill Factor: $48 \%$

Biased at $-32 \mathrm{~V}$ unless noted otherwise

NOTE: New "Red" version available with higher near-IR QE. NOT used in these tests. 


\section{Commercial transmitter $850 \mathrm{~nm}$ VCSEL (4 x 28G)}

\section{FINISAR}

Optical Transceivers 100GBASE-SR4 and OT FTLC9141SENM

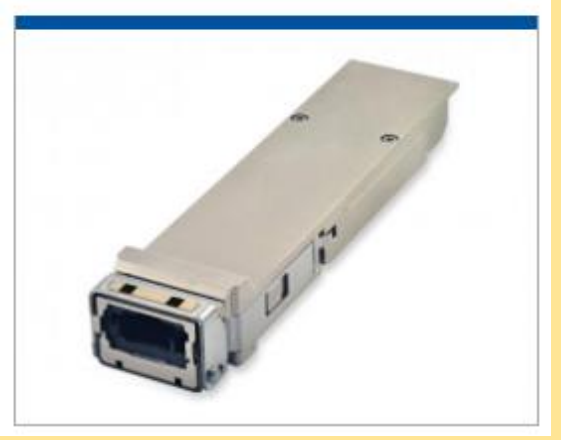

\section{Distance:}

Data Rate (max):

Protocol:

Low End Case Temperature $\left({ }^{\circ} \mathrm{C}\right)$ :

High End Case Temperature $\left({ }^{\circ} \mathrm{C}\right)$ :

Diagnostics:

Transmitter.

Receiver.

Voltage Supply:

Connector:

Wavelength:
$100 \mathrm{~m}$

$112 \mathrm{~Gb} / \mathrm{s}$

OTN OTU4 Compliant, 100G Ethernet Compliant $-5$

75

Digital

$4 \mathrm{x}$ VCSEL Array

PIN

3.3

MPO (MTP12)

$850 \mathrm{~nm}$ Band

We used 10G version in this work. 


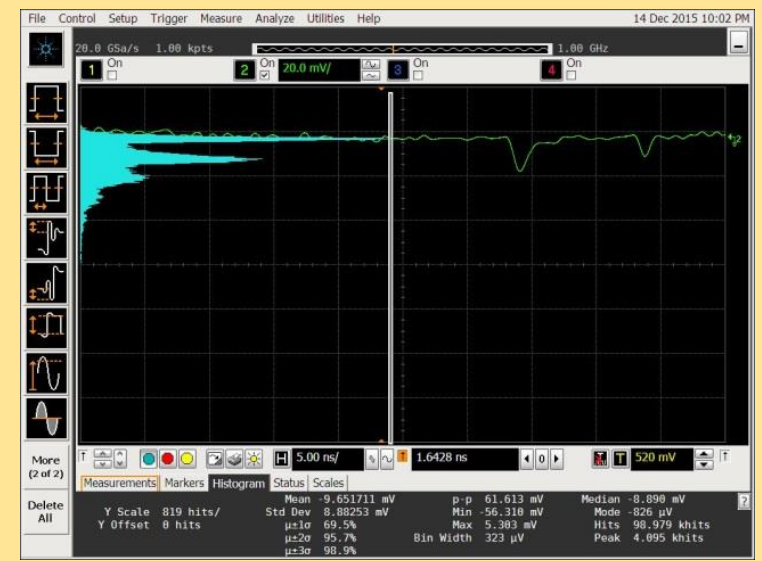

$\lambda=0.8$

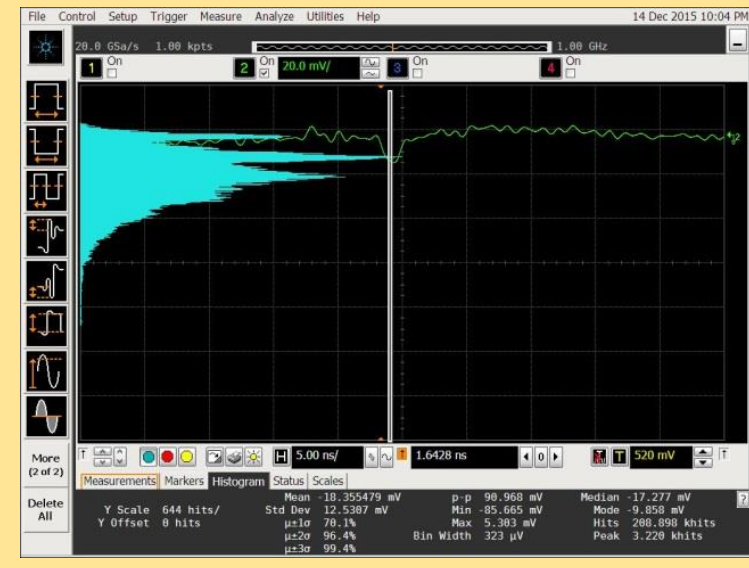

$\lambda=1.7$

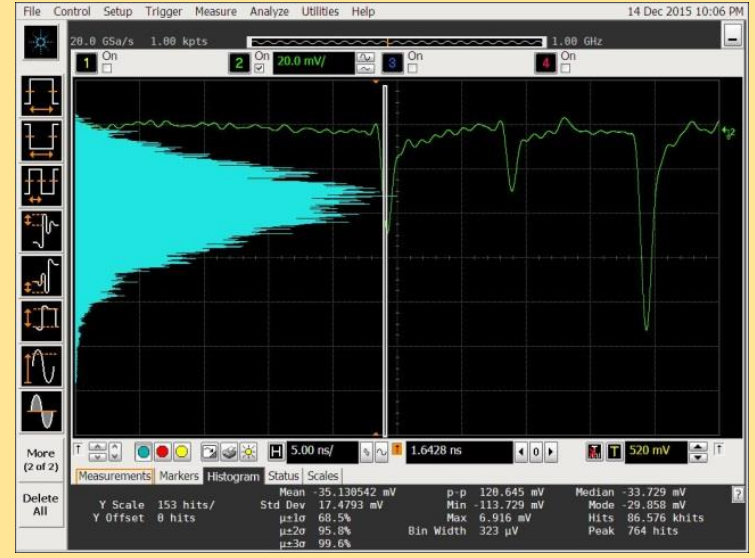

$\lambda=3.2$ 
Free-space optical communication bench test diagram for the Sensl APD arrays using coincidence detection.

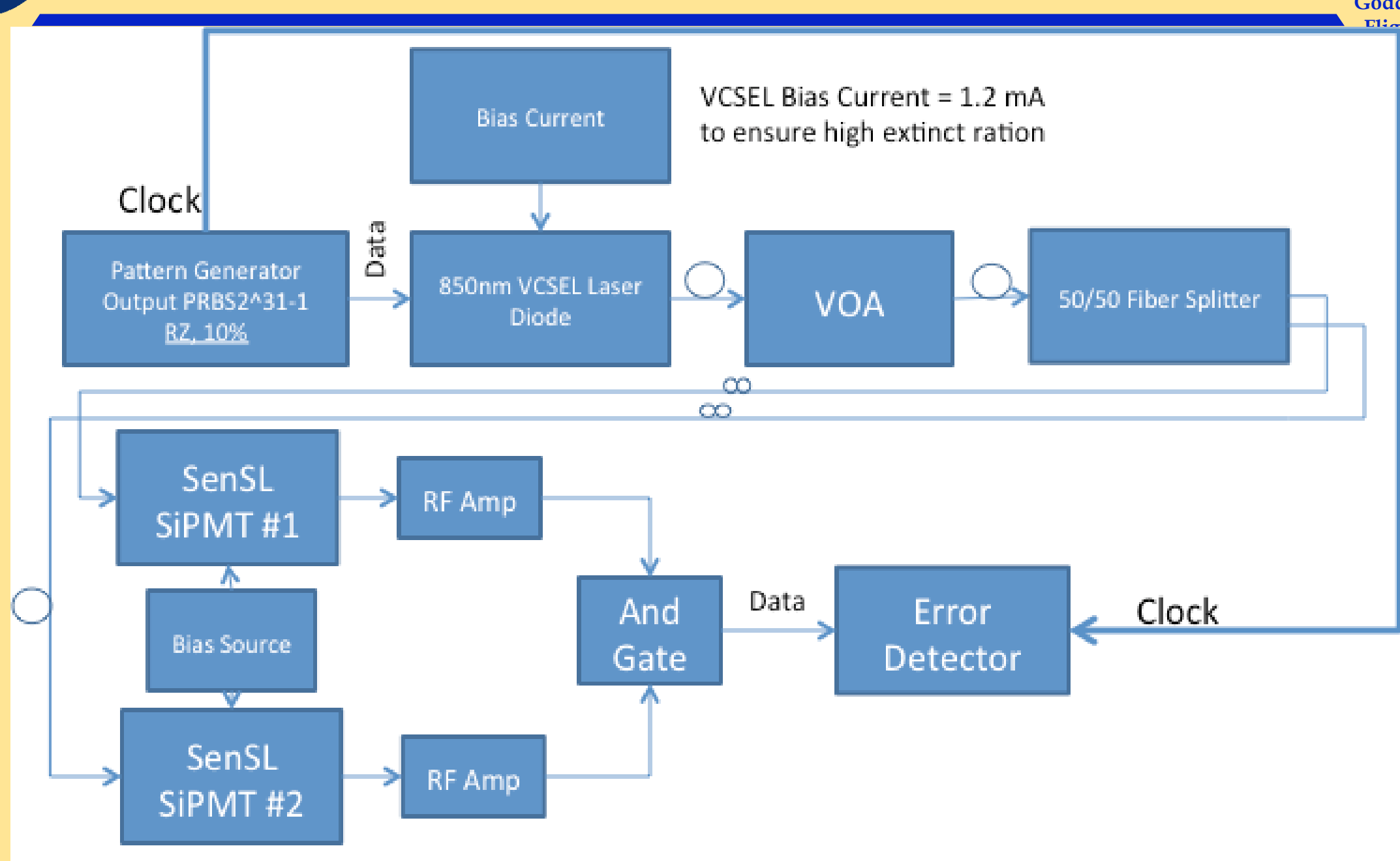

Sensel Bias Voltage $=32 \mathrm{~V}$ 


\section{Sensl APD array}

\section{Communication performance (@850 nm)}

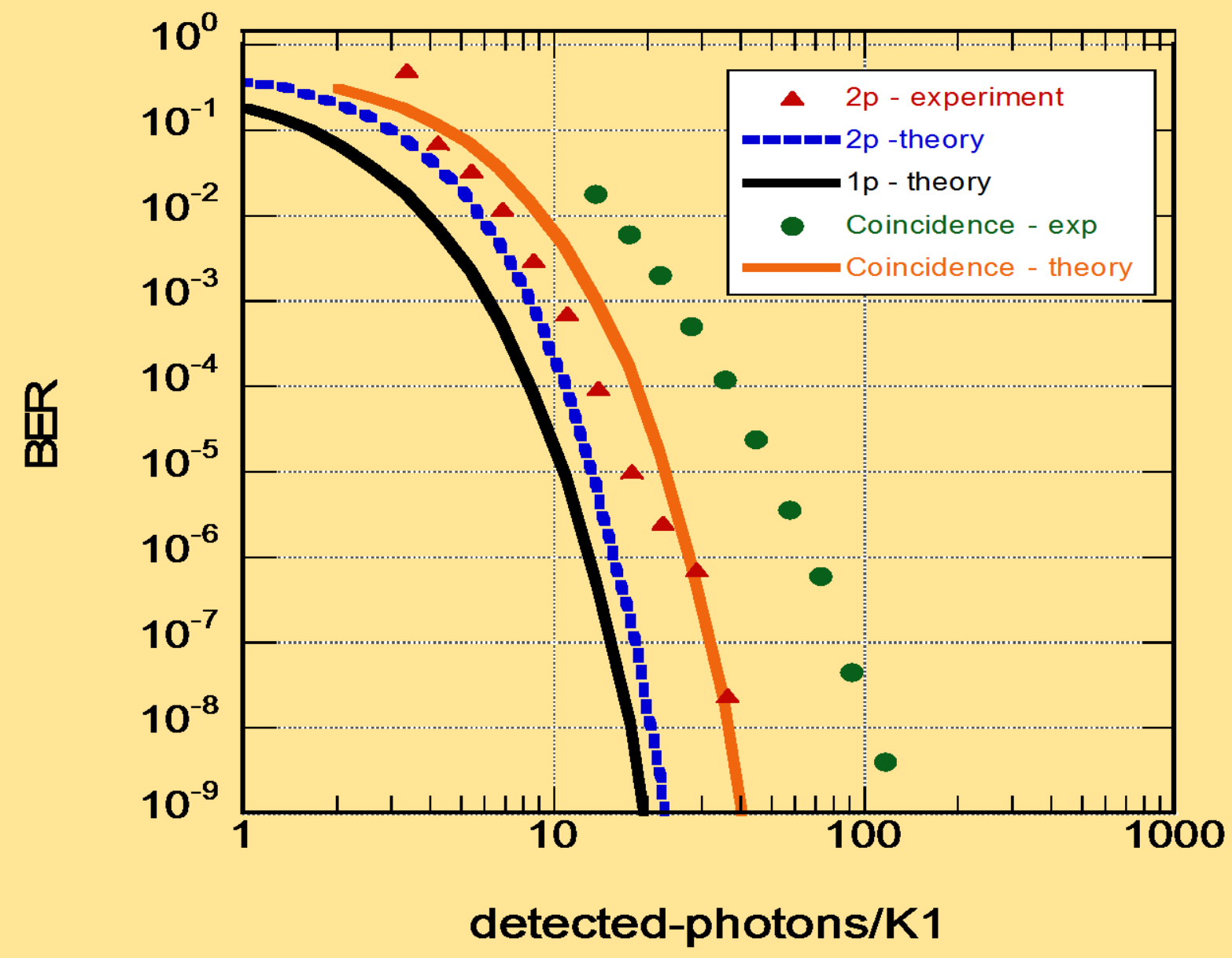

$\mathrm{RZ}-\mathrm{OOK} 100 \mathrm{Mbps}$ data rate with $\mathrm{PRBS}=2^{\wedge} 31-1$ 


\section{Sensl APD array}

Communication performance with coincidence detection at various rates

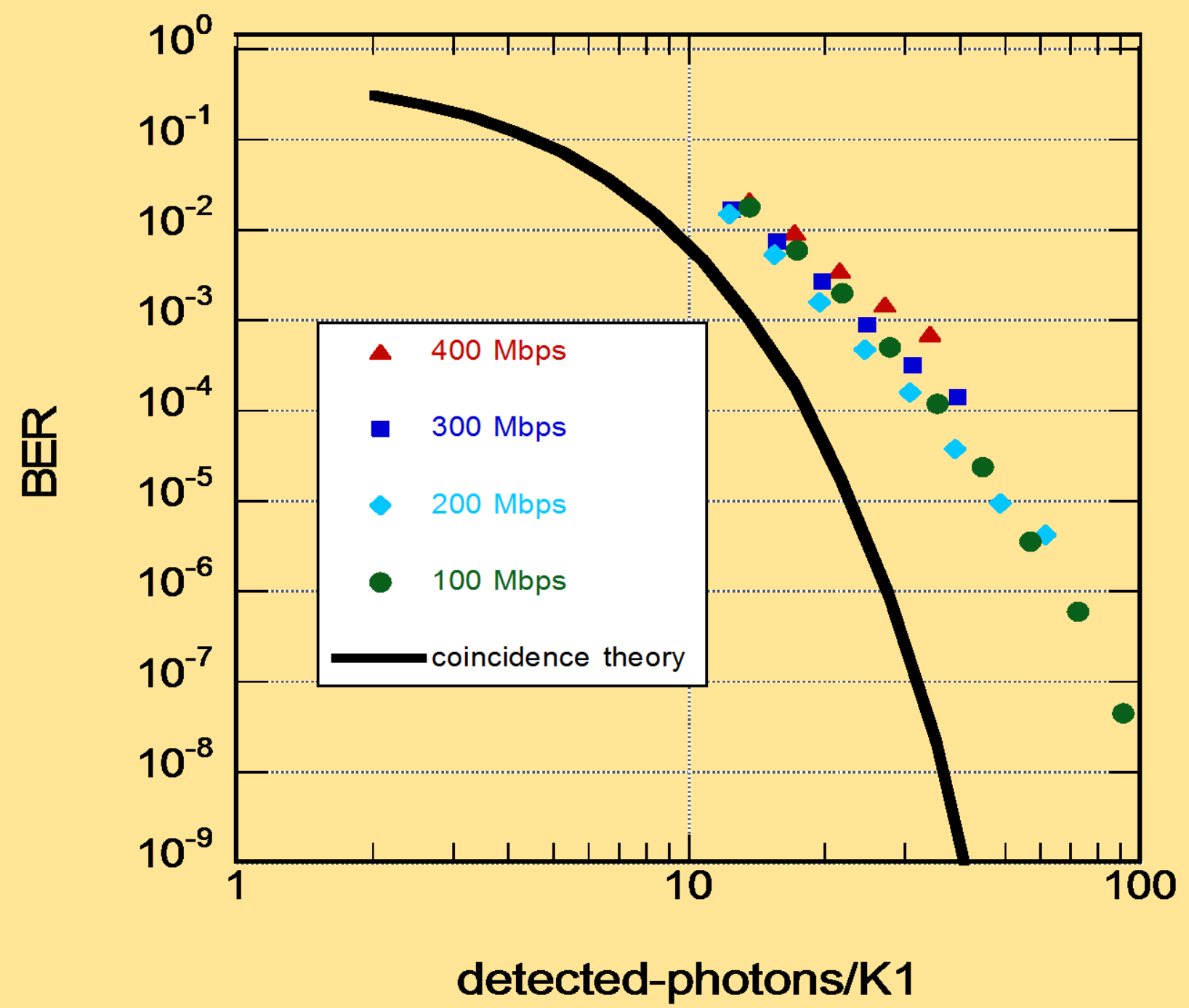




\section{DRS Inc. HgCdTe APD}
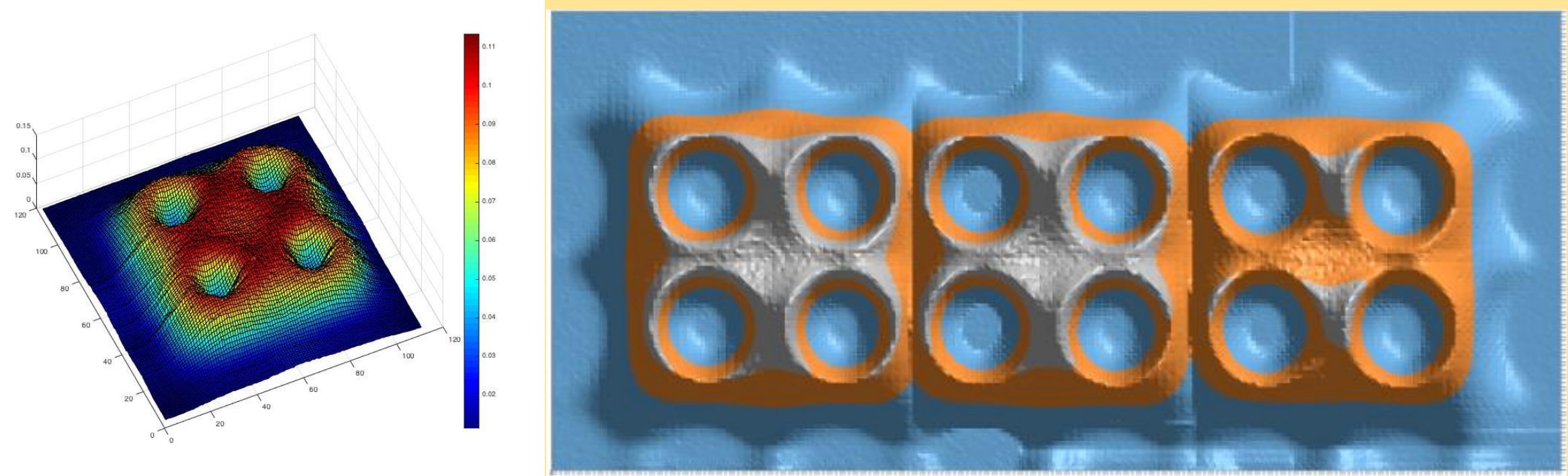

single pixel consisting of

3 pixels

4 mesas with $11 \mathrm{~V}$ 


\section{DRS HgCdTe APD \\ Intensity Pulse Height Distribution}

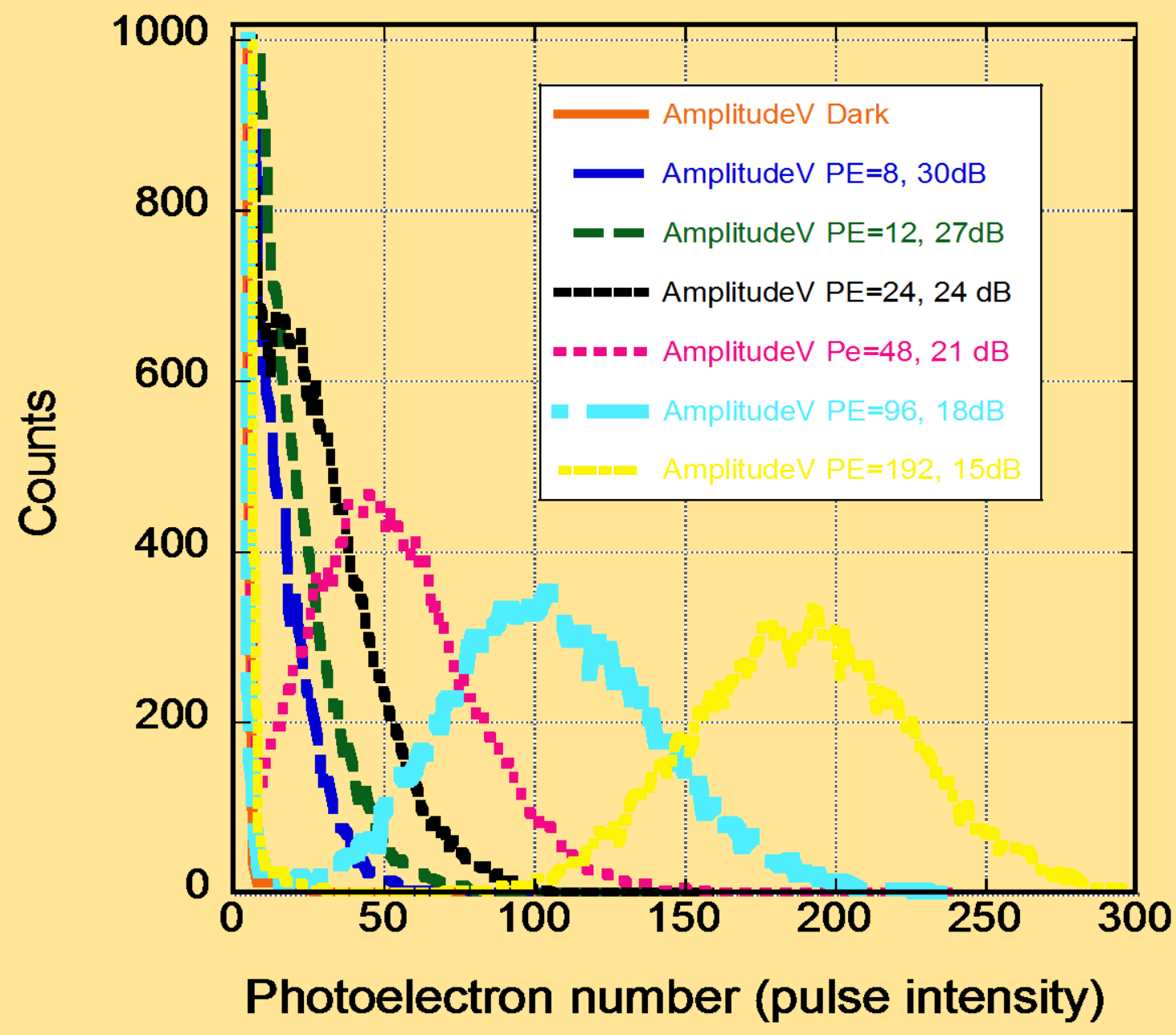




\section{Communication performance (@1550 nm) test with DRS HgCdTe APD receiver}

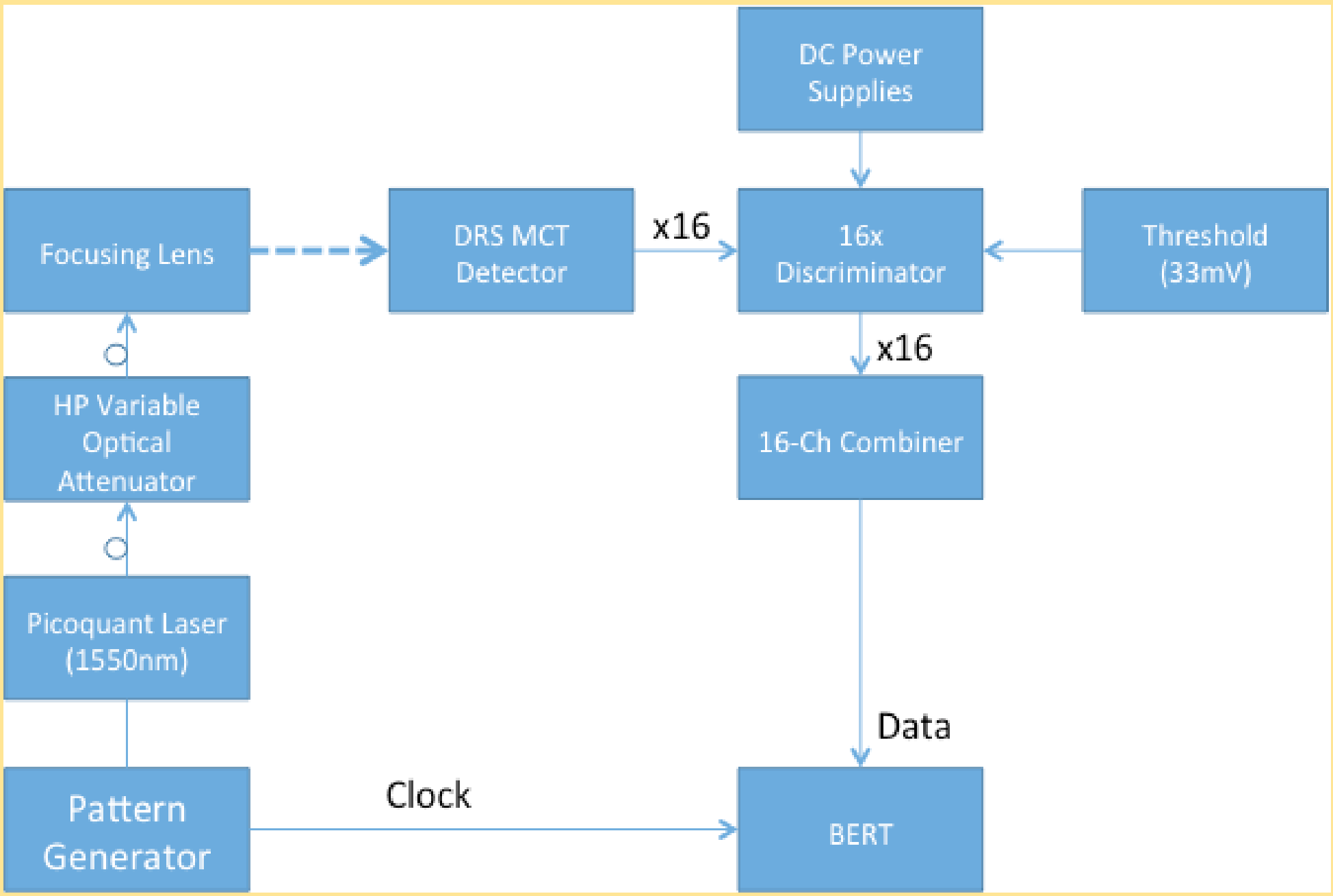


DRS HgCdTe APD experimental BER data from a single pixel

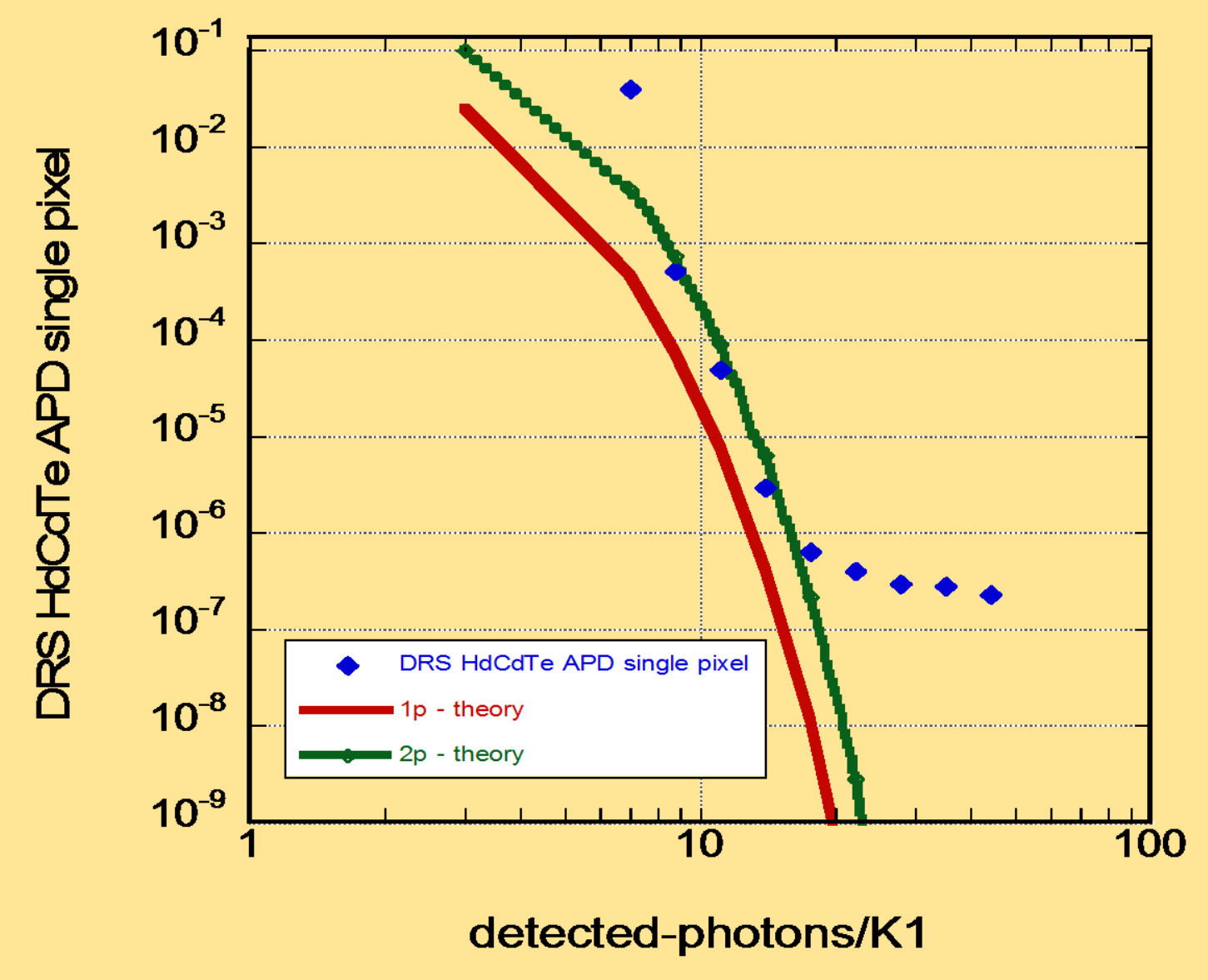

$\mathrm{RZ}-\mathrm{OOK} 50 \mathrm{Mbps}$ data rate with $\mathrm{PRBS}=2^{\wedge} 31-1$ 


\section{Novel photon-counting detectors for free-space communication SUMMARY}

I. Demonstrated photon-counting communication using three ideas:

1) use an array of photon-counting elements wired together as a single detector

2) use a high pass filter, ideally on each array element (or on the array output to only preserve the information-bearing portion of the waveform)*

3) depending on the photon-counting element excess noise, use either a "two-photon" intensity threshold level or an AND-gate with coincidence detection.

* $\quad$ Pavlov, N. "Silicon Photomultiplier and Readout Method" USPTO Patent Application Publication, No. US2013/0099100 A1 (2013).

II. Commercial components

Commercial $850 \mathrm{~nm}$ VCSEL transmitters and silicon APD Geiger-mode arrays provide a viable path to low-cost high-rate (500 Mbps) free-space optical communication links.

III. HgCdTe APD

Demonstrated excellent communication performance at 50 Mbps @ $1550 \mathrm{~nm}$ with single-pixel HgCdTe APD.

IV. Future

$>1$ Gbps with single array (in InGaAs) and multi-Gbps with WDM should be viable. 